\title{
Recreational Cannabis Use and Risk of Prescription Opioid Overdose: Insights from Pediatric Inpatients
}

Amaya Pankaj ${ }^{1}$, Kosisochukwu Oraka ${ }^{2}$, Emmanuelle J. Caraballo-Rivera ${ }^{3}$, Munazza Ahmad ${ }^{4}$, Shaheer Zahid $^{5}$, Sadaf Munir ${ }^{6}$, Gayathri Gurumurthy ${ }^{7}$, Onose Okoeguale ${ }^{8}$, Shikha Verma ${ }^{9}$, Rikinkumar S. Patel 10,11

1. Pediatric Medicine, Jawaharlal Institute of Postgraduate Medical Education and Research, Puducherry, IND 2. Medicine, Vinnytsia National Medical University, Vinnytsia, UKR 3. Medical Sciences, Ponce Health Sciences University, Ponce, PRI 4. Medicine, Lahore Medical \& Dental College, Lahore, PAK 5. Psychiatry, Saint James School of Medicine, Park Ridge, USA 6. Psychiatry, Jersey Shore University Medical Center, Neptune, USA 7. Internal Medicine, Shadan Institute of Medical Sciences, Hyderabad, IND 8. Psychiatry, Vinnytsia National Medical University, Vinnytsia, UKR 9. Psychiatry and Behavioral Sciences, Rogers Behavioral Health, Kenosha, USA 10. Psychiatry and Behavioral Sciences, Oklahoma State University, Tulsa, USA 11. Psychiatry, Griffin Memorial Hospital, Norman, USA

Corresponding author: Rikinkumar S. Patel, rpatel_09@arcadia.edu

\section{Abstract \\ Objectives}

Our first goal is to evaluate the prevalence of hospital admissions for prescription opioid overdose (POD) in pediatric inpatients, and next goal is to measure the independent association between cannabis use disorders (CUD) and POD.

\section{Methods}

We used the nationwide inpatient sample (NIS) and included $27,444,239$ pediatric inpatients (age $\leqslant 18$ years), and $10,562(0.04 \%)$ were managed primarily for POD. The odds ratio (OR) of the association of variables in POD inpatients was measured using the binomial logistic regression model that was adjusted for demographic confounders and psychiatric comorbidities.

\section{Results}

Adolescents have higher odds (OR 10.75, 95\% CI 10.16-11.36) of POD-related hospitalization compared to children $\leqslant 12$ years. Whites formed the significant proportion (67\%), and those from low-income families $(<50$ th percentile) had higher likelihood for POD-related hospitalization. The most prevalent psychiatric comorbidities were mood disorders (44.3\%) and anxiety disorders (14.6\%). Prevalent comorbid substance use disorders (SUDs) included cannabis (14.2\%), tobacco (13.1\%), and opioid (9.4\%). A higher odds of association with POD-related hospitalizations were seen in pediatric inpatients with comorbid opioid (OR 8.79, 95\% CI 8.08-9.56), tobacco (OR 1.58, 95\% CI 1.47-1.70), and cannabis (OR 1.68, 95\% CI 1.57-1.81) use disorders.

Review began 10/03/2020 Review ended 10/12/2020 Published 10/20/2020

๑) Copyright 2020

Pankaj et al. This is an open access article distributed under the terms of the Creative Commons Attribution License CC-BY 4.0., which permits unrestricted use, distribution, and reproduction in any medium, provided the original author and source are credited.

\section{Conclusion}

The prescription opioid is a bridge to opioid abuse/dependence, thereby increasing the risk of other SUDs like tobacco (by 58\%) and cannabis (by 68\%). Regulating the easy availability of prescription opioids and also improving the existing prescription trends are an essential way to reduce this problem. Finally, awareness and counseling are recommended strategies for harm reduction/rehabilitation among the pediatric population.

Categories: Pediatrics, Psychiatry, Epidemiology/Public Health

Keywords: prescription opioid, opioid overdose, adolescents, substance use, cannabis use, marijuana use, opioid use disorders

\section{Introduction}

A widespread emphasis and discussion over adequate assessment and treatment of pain in children are, over the past decade, resulting in a surge in the prescription of opioids in pediatric emergency departments (ED). Concurrently, opioid overdose-related hospitalizations and the non-medical use of opioids among adolescents have been a growing health concern [1]. The tendency of adolescents to engage in high-risk behavior and experimentation must be taken into account as substance and alcohol abuse, and violence tend to co-occur [2]. According to the national survey on drug use and health, prescription analgesics were the second most commonly abused substances by adolescents and young adults [1].

The opioid prescription seems to be influenced by factors like race, gender, and insurance. This trend mimics the adult population in terms that black patients seem to receive fewer opioid prescriptions, a trend which may be attributed to lack of access to medical care and medications due to cost and lesser availability of pharmacy $[3,4]$. Thereby, white children seem to form a sizable proportion of the opioid-related hospitalizations. Also, there is an increased tendency to prescribe opioids in patients on private insurance, 
which may be due to improving patient satisfaction, but again this exposes the child to easier availability of opioids for misuse [5]. Though pain assessment and relief are of utmost importance in the pediatric population, studies have shown that inadequate pain relief in this age group can have a worse prognosis, but also making easier availability of opioids can lead to accidental prescription opioid overdoses, poisoning, and misuse, which should be given greater attention [6,7].

We used the largest inpatient database from the United States (US) hospitals to evaluate the prevalence of hospital admissions for prescription opioid overdose (POD) in pediatric inpatients and to measure the independent association between cannabis use disorders (CUD) and POD-related hospitalizations. Also, we studied the differences in demographic patterns and comorbid psychiatric and substance use disorders (SUDs) for POD with CUD versus without CUD.

\section{Materials And Methods}

\section{Data source}

We used the nationwide inpatient sample (NIS) from the healthcare cost and utilization project (HCUP), which is the largest hospital-based dataset in the United States. The NIS constitutes patient discharge diagnosis information (clinical and non-clinical) from 4,400 hospitals across the 44 states in the Unted States. Diagnostic information in the NIS is identified using the international classification of diseases, ninth edition (ICD-9) codes [8].

\section{Inclusion criteria}

We conducted a retrospective analysis of the NIS (2010 to 2014) to identify all non-elective/emergency admissions for pediatric patients (age 18 and below) with a primary ICD-9 discharge diagnosis for POD (965.00, 95.01, and 965.09). These patients were further grouped by comorbid CUD identified by ICD-9 codes (304.30, 304.31, 304.32, 305.20, 305.21, and 305.22). Patients coded as "in remission" from cannabis abuse or dependence were excluded from our analysis to maintain the uniformity of the study cohort.

\section{Variables}

Demographic variables including age, sex, race, and median household income were evaluated in this study. Psychiatric comorbidities (anxiety disorder, mood disorders, psychotic disorders, and suicidality) and SUDs (opioid, amphetamine, and cocaine) were identified using the ICD-9 diagnosis codes [9].

\section{Statistical analysis}

The analyses were conducted using the statistical package for the social sciences (SPSS) version 26 (IBM Corp., Armonk, NY). We utilized bivariate descriptive analysis to compare demographics and psychiatric comorbidities and SUDs in patients with POD and without POD (non-POD) cohorts followed by binomial logistic regression analyses to evaluate the demographic predictors and risk factors for POD-related hospitalization. Next, we evaluated the age-wise odds for POD-related hospitalization in patients with CUD compared to patients without CUD (non-CUD). The regression models were adjusted for demographics confounders (age, sex, race, and median household income) and psychiatric comorbidities. Lastly, we compared CUD and non-CUD cohorts using bivariate descriptive analysis followed by binomial logistic regression analyses to identify the demographic predictors and risk factors in POD patients with CUD. Due to the large sample size and multiple comparisons of data, statistical significance was set at $\mathrm{p}$ value $<0.01$.

\section{Ethical approval}

Unique subject identifiers were used to secure patient information. The use of the publicly available deidentified NIS data of the HCUP, according to the department of health and human services does not require approval from an institutional review board [8].

\section{Results}

We analyzed a total of 27,444,239 pediatric hospitalizations, and 10,562 (0.04\%) were primarily POD-related hospitalization. Adolescents have 11 times higher odds (95\% CI 10.16-11.36) of POD-related hospitalization compared to children under 11 years, and whites form about two-third proportion (67\%) of the POD-cohort. POD-related hospitalizations were seen in children from low- to middle-income families by 1.3 times compared to those from high-income families (above 75 th percentile).

The most prevalent psychiatric comorbidities were mood disorders and anxiety disorders, and children diagnosed with mood disorders had 4.6 times higher odds (95\% CI 4.40-4.92) of POD-related hospitalization. The most prevalent comorbid SUDs include cannabis (14.2\%), tobacco (13.1\%), and opioid (9.4\%) use disorders. There exists a higher odds of association with POD-related hospitalizations in children with comorbid opioid (OR 8.79, 95\% CI 8.08-9.56), tobacco (OR 1.58, 95\% CI 1.47-1.70), and cannabis (OR 1.68, 95\% CI 1.57-1.81) use disorders as shown in Table 1. 


\begin{tabular}{|c|c|c|c|c|c|}
\hline Variable & Non-POD (\%) & POD (\%) & Odds ratio & 95\% Confidence interval & $p$ value \\
\hline Total inpatients & $27,433,677$ & 10,562 & - & - & - \\
\hline \multicolumn{6}{|l|}{ Age at admission } \\
\hline$<11$ years & 88.9 & 26.6 & Reference & & \\
\hline $12-18$ years & 11.1 & 73.4 & 10.75 & $10.16-11.36$ & $<0.001$ \\
\hline \multicolumn{6}{|l|}{ Sex } \\
\hline Male & 51.0 & 48.0 & Reference & & \\
\hline Female & 49.0 & 52.0 & 0.87 & $0.83-0.91$ & $<0.001$ \\
\hline \multicolumn{6}{|l|}{ Race } \\
\hline White & 51.3 & 67.0 & Reference & & \\
\hline Black & 15.8 & 12.4 & 0.61 & $0.57-0.65$ & $<0.001$ \\
\hline Hispanic & 21.4 & 12.5 & 0.59 & $0.55-0.63$ & $<0.001$ \\
\hline Other & 11.5 & 8.0 & 0.84 & $0.77-0.91$ & $<0.001$ \\
\hline \multicolumn{6}{|c|}{ Median household income } \\
\hline $0-25^{\text {th }}$ percentile & 29.0 & 28.8 & 1.29 & $1.21-1.37$ & $<0.001$ \\
\hline $26^{\mathrm{th}}-50^{\mathrm{th}}$ percentile & 25.1 & 27.1 & 1.35 & $1.27-1.44$ & $<0.001$ \\
\hline $51^{\mathrm{st}}-75^{\text {th }}$ percentile & 24.6 & 25.2 & 1.29 & $1.21-1.37$ & $<0.001$ \\
\hline $76^{\mathrm{n}}-100^{\mathrm{tn}}$ percentile & 21.4 & 18.9 & Reference & & \\
\hline \multicolumn{6}{|c|}{ Comorbid psychiatric disorders } \\
\hline Anxiety disorders & 1.4 & 14.6 & 1.03 & $0.97-1.10$ & 0.339 \\
\hline Mood disorders & 3.0 & 44.3 & 4.65 & $4.40-4.92$ & $<0.001$ \\
\hline Suicidality & 1.0 & 7.1 & 0.41 & $0.38-0.45$ & $<0.001$ \\
\hline Psychotic disorders & 0.3 & 1.6 & 0.73 & $0.62-0.86$ & $<0.001$ \\
\hline \multicolumn{6}{|c|}{ Comorbid substance use disorders } \\
\hline Tobacco & 0.6 & 13.1 & 1.58 & $1.47-1.70$ & $<0.001$ \\
\hline Cannabis & 0.6 & 14.2 & 1.68 & $1.57-1.81$ & $<0.001$ \\
\hline Opioid & 0.1 & 9.4 & 8.79 & $8.08-9.56$ & $<0.001$ \\
\hline Amphetamine & 0 & 1.0 & 0.66 & $0.53-0.82$ & $<0.001$ \\
\hline Cocaine & 0 & 1.5 & 0.83 & $0.69-0.99$ & 0.043 \\
\hline
\end{tabular}

\section{TABLE 1: Suspected risk factors for prescription opioid overdose-related hospitalization.}

The proportion of non-POD and POD patients was obtained using cross-tabulation, and the odds ratio was generated by the binomial logistic regression model and was adjusted for all variables mentioned.

POD: Prescription opioid overdose. 


\begin{tabular}{|c|c|c|c|c|c|}
\hline Variable & No CUD (\%) & CUD (\%) & Odds ratio & $95 \%$ Confidence interval & $\mathrm{p}$ value \\
\hline Total inpatients & 9,058 & 1,505 & - & - & - \\
\hline \multicolumn{6}{|l|}{ Age at admission } \\
\hline$<11$ years & 29.7 & 7.8 & Reference & & \\
\hline 12-18 years & 70.3 & 92.2 & 1.96 & $1.56-2.44$ & $<0.001$ \\
\hline \multicolumn{6}{|l|}{ Sex } \\
\hline Male & 46.0 & 59.9 & Reference & & \\
\hline Female & 54.0 & 40.1 & 0.55 & $0.48-0.63$ & $<0.001$ \\
\hline \multicolumn{6}{|l|}{ Race } \\
\hline White & 65.6 & 75.2 & Reference & & \\
\hline Black & 13.5 & 6.4 & 0.66 & $0.51-0.85$ & 0.001 \\
\hline Hispanic & 12.7 & 11.3 & 1.14 & $0.92-1.40$ & 0.225 \\
\hline Other & 8.2 & 7.1 & 0.82 & $0.62-1.07$ & 0.135 \\
\hline \multicolumn{6}{|c|}{ Median household income } \\
\hline $0-25^{\text {th }}$ percentile & 29.1 & 26.5 & 0.83 & $0.69-1.01$ & 0.064 \\
\hline $26^{\text {th }}-50^{\text {th }}$ percentile & 27.5 & 25.0 & 0.82 & $0.68-0.99$ & 0.040 \\
\hline $51^{\text {st }}-75^{\text {th }}$ percentile & 25.2 & 25.1 & 0.82 & $0.68-0.99$ & 0.034 \\
\hline $76^{\text {th }}-100^{\text {th }}$ percentile & 18.1 & 23.4 & Reference & & \\
\hline \multicolumn{6}{|c|}{ Comorbid psychiatric disorders } \\
\hline Anxiety disorders & 13.7 & 20.3 & 1.13 & $0.95-1.33$ & 0.174 \\
\hline Mood disorders & 41.9 & 58.7 & 1.77 & $1.53-2.05$ & $<0.001$ \\
\hline Suicidality & 6.8 & 8.9 & 1.21 & $0.97-1.52$ & 0.097 \\
\hline Psychotic disorders & 1.4 & 2.7 & 1.34 & $0.88-2.04$ & 0.175 \\
\hline \multicolumn{6}{|c|}{ Comorbid substance use disorders } \\
\hline Tobacco & 9.6 & 33.7 & 3.01 & $2.58-3.51$ & $<0.001$ \\
\hline Opioid & 6.7 & 25.4 & 2.78 & $2.35-3.30$ & $<0.001$ \\
\hline Amphetamine & 0.4 & 4.6 & 5.79 & $3.64-9.23$ & $<0.001$ \\
\hline Cocaine & 0.7 & 6.9 & 6.99 & $4.76-10.29$ & $<0.001$ \\
\hline
\end{tabular}

TABLE 2: Suspected risk factors for cannabis use disorders in children with prescription opioid overdose.

The proportion of non-CUD and CUD patients was obtained using cross-tabulation, and the odds ratio was generated by the binomial logistic regression model and was adjusted for all variables mentioned.

CUD: Cannabis use disorders.

\section{Discussion}

In overall pediatric hospitalizations, the prevalence of POD was $0.04 \%$ and majorly in adolescents and whites. About, half of the POD-related hospitalizations had mood disorders, and the most prevalent substance used was cannabis (14.2\%) and tobacco (13.1\%). After controlling for potential confounders, CUD was associated with $68 \%$ increased odds for POD-related hospitalization.

Adolescents were at higher risk of POD-related hospitalizations compared to children under 11 years. This was consistent with findings of a study using the national poison data system (NPDS), which found that prescription opioid exposure was highest among teenagers [10]. This may be attributable to the thrillseeking behavior, peer pressure, and accessibility to prescription opioids, which doubled the rates of POD 
among adolescents and young adults since 1994 [11]. Whites constituted about 67\% of total POD-related hospitalizations in our study, which is possible as they may have greater access and probability of receiving prescription opioids during ED visits compared to minorities [12]. Another notable finding was that PODrelated hospitalizations were higher in low- to middle-income families compared to those from high-income families, and a similar trend was seen in the adult population $[7,13]$. This may be attributable to the easier availability and cheaper cost of prescription opioids relative to other substances with abuse potential. Males seem to have a slightly higher risk than females, but the difference is not as stark as with other illicit substances, which may be due to the fact that females are more likely to be prescribed an opioid and also the perception that abusing prescription medication is "safer" than abuse of other substances [14].

Our study found that there is a higher risk of psychiatric comorbidities in pediatric inpatients with POD. The most common psychiatric conditions associated are mood disorders and anxiety disorders, which are consistent with the study using the data from the national epidemiologic study on alcohol and related conditions (NESARC). This explains the bidirectional association between POD and psychiatric comorbidities, where adolescents tend to use prescription opioids to alleviate their mood symptoms. We also found that POD-related hospitalizations had comorbid SUDs with opioids, cannabis, and tobacco, with cannabis being the most prevalent substance used due to the rising use of cannabis among the pediatric population [15]. Also, an inpatient study found a higher prevalence of tobacco (36.8\%) and cannabis (28.5\%) use disorders among pediatric patients hospitalized for heroin overdoses [16].

There was a unique trend in risk factors, epidemiological outcomes, psychiatric comorbidities, and SUDs in the pediatric inpatients with CUD among POD-related hospitalizations. CUD was majorly seen in adolescents and males, which may be due to the reduced perception of risk and easier access to cannabis [17]. Though data from the national surveys on drug use and health suggests blacks form the majority having CUD in adults, in our study white children formed about three-fourths of POD-related hospitalizations. Yet, when compared with whites, hispanics with POD had 14\% higher odds of comorbid CUD [18]. Pediatric inpatients with POD from high-income families were more likely to have CUD. This is in accordance with a study by Trim et al., which found that adolescents living in an affluent neighborhood are more likely to abuse substances due to lesser supervision [19].

Among cannabis users, there exists a significantly high risk of comorbid SUDs with higher odds of using cocaine (by seven times), amphetamines (by six times), and tobacco and opioids (by three times each) $[20,21]$. Mood and anxiety disorders, suicidal behaviors, and psychotic disorders were significantly associated with pediatric POD inpatients having CUD [22]. Youth with CUD at an earlier age have a greater risk of long-lasting cognitive impairment and several psychiatric and medical conditions [23-28].

Regulating the easy availability of prescription opioids and also improving the existing prescription trends is an essential way to reduce the problem of the current opioid epidemic in the United States. In the current opioid crisis, there is improved vigilance on prescribed opioids as compared to before, and the overall number of prescribed opioids is trending down. It is important that providers continue to regulate prescribed opioids. Parents and guardians should also understand the importance of safe storage of prescriptions, thereby keeping the medications out of reach from children [29]. It is imminent to engage youth and families in psychoeducation, harm reduction strategies, services provided in the community to drop extra medications, and if there are concerns for opioid abuse/dependence, providing individuals and families with naloxone spray. Earlier onset of SUDs is associated with long-term implications such as declining functioning due to ongoing financial, social, and occupational challenges and continued mental health and SUDs. The United States has the largest rates of an opioid burden for both males (968.6 disability-adjusted life years [DALYs] per 100,000) and females (565.7 DALYs per 100,000), and years of life lost (YLL) is also high at 265 per $100,000[30]$.

There were a few limitations to our study. First, these findings may be underreported, thereby making it unrepresentative of the real burden of POD-related hospitalizations across the United States due to the nature of an administrative database that was used in this study. Second, we examined pooled data and year-by-year trend data, due to which we cannot rule out the possibility that outpatients and rehospitalizations across different healthcare settings may not be represented. We used the NIS that enabled us to get a national representative sample of POD inpatients, and the large sample size also increased the power of our study. We analyzed trends of comorbid CUD in POD inpatients, which gave us a unique picture and increased external validity. Also, the findings of this study are relevant to understand the burden of POD, which can help to adequately understand and strategically devise specific intervention plans to control this public health problem.

\section{Conclusions}

POD-related hospitalizations were observed to have a higher prevalence among white adolescents from affluent families. There are increased risks for other coexisting SUDs, most commonly with CUD and mental health concerns such as anxiety and mood disorders. Decreasing prescription opioids, parental education, youth awareness, understanding, and counseling are the recommended strategies for harm reduction and rehabilitation among the at-risk pediatric populations. Continued opioid abuse/dependence is associated with continued psychiatric and substance use challenges in adulthood. Opioid abuse/dependence is related to increased disease burden causing high DALYs and YLLs in the United States. Regulating the easy availability of prescription opioids and also improving the existing prescription trends are an essential way to reduce this problem. 


\section{Additional Information \\ Disclosures}

Human subjects: Consent was obtained by all participants in this study. Animal subjects: All authors have confirmed that this study did not involve animal subjects or tissue. Conflicts of interest: In compliance with the ICMJE uniform disclosure form, all authors declare the following: Payment/services info: All authors have declared that no financial support was received from any organization for the submitted work. Financial relationships: All authors have declared that they have no financial relationships at present or within the previous three years with any organizations that might have an interest in the submitted work. Other relationships: All authors have declared that there are no other relationships or activities that could appear to have influenced the submitted work.

\section{References}

1. Mazer-Amirshahi M, Mullins PM, Rasooly IR, van den Anker J, Pines JM: Trends in prescription opioid use in pediatric emergency department patients. Pediatr Emerg Care. 2014, 30:230-235. 10.1097/PEC.0000000000000102

2. Whiteside LK, Walton MA, Bohnert AS, Blow FC, Bonar EE, Ehrlich P, Cunningham RM: Nonmedical prescription opioid and sedative use among adolescents in the emergency department. Pediatrics. 2013, 132:825-832. 10.1542/peds.2013-0721

3. Anand KJ, Willson DF, Berger J, et al.: Tolerance and withdrawal from prolonged opioid use in critically ill children. Pediatrics. 2010, 125:e1208-e1225. 10.1542/peds.2009-0489

4. Playfor S, Jenkins I, Boyles C, et al.: Consensus guidelines on sedation and analgesia in critically ill children . Intensive Care Med. 2006, 32:1125-1136. 10.1007/s00134-006-0190-x

5. Tamayo-Sarver JH, Hinze SW, Cydulka RK, Baker DW: Racial and ethnic disparities in emergency department analgesic prescription. Am J Public Health. 2003, 93:2067-2073. 10.2105/ajph.93.12.2067

6. Yaster M, Deshpande JK: Management of pediatric pain with opioid analgesics . J Pediatr. 1988, 113:421-429. 10.1016/s0022-3476(88)80622-X

7. Cerda M, Gaidus A, Keyes KM, Ponicki W, Martins S, Galea S, Gruenewald P: Prescription opioid poisoning across urban and rural areas: identifying vulnerable groups and geographic areas. Addiction. 2017, 112:103112. 10.1111/add.13543

8. NIS database documentation. (2019). Accessed: Aug 1, 2020: https://www.hcupus.ahrq.gov/db/nation/nis/nisdbdocumentation.jsp.

9. NIS description of data elements. (2019). Accessed: Aug 1, 2020: https://www.hcupus.ahrq.gov/db/nation/nis/nisdde.jsp.

10. Allen ID, Casavant MJ, Spiller HA, Chounthirath T, Hodges NL, Smith GA: Prescription opioid exposures among children and adolescents in the United States: 2000-2015. Pediatrics. 2017, 139:e20163382. 10.1542/peds.2016-3382

11. McCabe SE, West BT, Teter CJ, Boyd CJ: Medical and nonmedical use of prescription opioids among high school seniors in the United States. Arch Pediatr Adolesc Med. 2012, 166:797-802. 10.1001/archpediatrics.2012.85

12. Groenewald CB, Rabbitts JA, Hansen EE, Palermo TM: Racial differences in opioid prescribing for children in the United States. Pain. 2018, 159:2050-2057. 10.1097/j.pain.0000000000001290

13. The opioid crisis and economic opportunity: geographic and economic trends. (2018). Accessed: April 10, 2020: https://aspe.hhs.gov/system/files/pdf/259261/ASPEEconomicOpportunityOpioidCrisis.pdf.

14. McHugh RK, Nielsen S, Weiss RD: Prescription drug abuse: from epidemiology to public policy . J Subst Abuse Treat. 2015, 48:1-7. 10.1016/j.jsat.2014.08.004

15. Behavioral health trends in the United States: results from the 2014 national survey on drug use and health . (2015). Accessed: April 10, 2020: https://www.samhsa.gov/data/sites/default/files/NSDUH-FRR12014/NSDUH-FRR1-2014.pdf.

16. Queeneth U, Bhimanadham NN, Mainali P, Onyeaka HK, Pankaj A, Patel RS: Heroin overdose-related child and adolescent hospitalizations: insight on comorbid psychiatric and substance use disorders. Behav Sci (Basel). 2019, 9:77. 10.3390/bs9070077

17. Simpson AK, Magid V: Cannabis use disorder in adolescence. Child Adolesc Psychiatr Clin N Am. 2016, 25:431-443. 10.1016/i.chc.2016.03.003

18. Wu LT, Brady KT, Mannelli P, Killeen TK, Workgroup NA: Cannabis use disorders are comparatively prevalent among nonwhite racial/ethnic groups and adolescents: a national study. J Psychiatr Res. 2014, 50:26-35. 10.1016/j.jpsychires.2013.11.010

19. Trim RS, Chassin L: Neighborhood socioeconomic status effects on adolescent alcohol outcomes using growth models: exploring the role of parental alcoholism. J Stud Alcohol Drugs. 2008, 69:639-648 10.15288/jsad.2008.69.639

20. Hall W, Degenhardt L: Adverse health effects of non-medical cannabis use . Lancet. 2009, 374:1383-1391. 10.1016/S0140-6736(09)61037-0

21. Ruhm CJ: Drug involvement in fatal overdoses. SSM Popul Health. 2017, 3:219-226. 10.1016/i.ssmph.2017.01.009

22. The link between substance abuse, violence, and suicide. (2011). Accessed: April 15, 2020: https://www.psychiatrictimes.com/substance-use-disorder/link-between-substance-abuse-violence-andsuicide.

23. Use of medical marijuana in children and adolescents with autism spectrum disorder for core autism symptoms or co-occurring emotional or behavioral problems. (2019). Accessed: April 10, 2020: https://www.aacap.org/AACAP/Policy_Statements/2019/Use_of_Medical_Marijuana_in_Children_and_Adolescents_with_Autisn

24. Patel RS, Goyal H, Satodiya R, Tankersley WE: Relationship of cannabis use disorder and irritable bowel syndrome (ibs): an analysis of 6.8 million hospitalizations in the United States. Subst Use Misuse. 2020, 55:281-290. 10.1080/10826084.2019.1664591

25. Patel RS, Manocha P, Patel J, Patel R, Tankersley WE: Cannabis use is an independent predictor for acute myocardial infarction related hospitalization in younger population. J Adolesc Health. 2020, 66:79-85. 10.1016/j.jadohealth.2019.07.024 


\section{Cureus}

26. Jaladi PR, Patel V, Kuduva Rajan S, et al.: Arrhythmia-related hospitalization and comorbid cannabis use disorder: trend analysis in US hospitals (2010-2014). Cureus. 2019, 11:e5607. 10.7759/cureus.5607

27. Patel RS, Patel J, Jaladi PR, Bhimanadham NN, Imran S, Tankersley WE: Burden of persistent vomiting with cannabis use disorder: report from 55,549 hospitalizations in the United States. Psychosomatics. 2019, 60:549-555. 10.1016/j.psym.2019.07.003

28. Patel RS, Mekala HM, Tankersley WE: Cannabis use disorder and epilepsy: a cross-national analysis of 657 072 hospitalized patients. Am J Addict. 2019, 28:353-360. 10.1111/ajad.12898

29. Yule AM, Lyons RM, Wilens TE: Opioid use disorders in adolescents-updates in assessment and management. Curr Pediatr Rep. 2018, 6:99-106. 10.1007/s40124-018-0161-z

30. Orpana HM, Lang JJ, Baxi M, et al.: Canadian trends in opioid-related mortality and disability from opioid use disorder from 1990 to 2014 through the lens of the global burden of disease study. Health Promot Chronic Dis Prev Can. 2018, 38:234-243. 10.24095/hpcdp.38.6.03 